\title{
Genomic composition and evolution of Aedes aegypti chromosomes revealed by the analysis of physically mapped supercontigs
}

Vladimir A Timoshevskiy ${ }^{1}$, Nicholas A Kinney ${ }^{2}$, Becky S deBruyn ${ }^{3}$, Chunhong Mao ${ }^{4}$, Zhijian Tu ${ }^{5}$, David W Severson ${ }^{3}$, Igor V Sharakhov' ${ }^{1}$ and Maria V Sharakhova ${ }^{1 *}$

\begin{abstract}
Background: An initial comparative genomic study of the malaria vector Anopheles gambiae and the yellow fever mosquito Aedes aegypti revealed striking differences in the genome assembly size and in the abundance of transposable elements between the two species. However, the chromosome arms homology between An. gambiae and Ae. aegypti, as well as the distribution of genes and repetitive elements in chromosomes of Ae. aegypti, remained largely unexplored because of the lack of a detailed physical genome map for the yellow fever mosquito.

Results: Using a molecular landmark-guided fluorescent in situ hybridization approach, we mapped $624 \mathrm{Mb}$ of the Ae. aegypti genome to mitotic chromosomes. We used this map to analyze the distribution of genes, tandem repeats and transposable elements along the chromosomes and to explore the patterns of chromosome homology and rearrangements between Ae. aegypti and An. gambiae. The study demonstrated that the $q$ arm of the sex-determining chromosome 1 had the lowest gene content and the highest density of minisatellites.

A comparative genomic analysis with An. gambiae determined that the previously proposed whole-arm synteny is not fully preserved; a number of pericentric inversions have occurred between the two species. The sex-determining chromosome 1 had a higher rate of genome rearrangements than observed in autosomes 2 and 3 of Ae. aegypti.

Conclusions: The study developed a physical map of $45 \%$ of the Ae. aegypti genome and provided new insights into genomic composition and evolution of Ae. aegypti chromosomes. Our data suggest that minisatellites rather than transposable elements played a major role in rapid evolution of chromosome 1 in the Aedes lineage. The research tools and information generated by this study contribute to a more complete understanding of the genome organization and evolution in mosquitoes.
\end{abstract}

Keywords: Physical mapping, Mosquito, Genome, Chromosome

\section{Background}

The genome of the major vector of arboviruses Aedes aegypti [1] was the second published mosquito genome after the genome of the malaria vector Anopheles gambiae [2]. The size of Ae. aegypti genome - 1,376 mega base pairs $(\mathrm{Mb})$ - is the largest among other mosquito genomes sequenced so far. It is five times bigger than the $264 \mathrm{Mb}$ genome of An. gambiae. The most striking difference

\footnotetext{
* Correspondence: msharakh@vt.edu

'Department of Entomology, Fralin Life Science Institute, Virginia Tech, Blacksburg, VA, USA

Full list of author information is available at the end of the article
}

between these two mosquito genomes is in the abundance of transposable elements (TEs). TEs cover approximately $50 \%$ of the Ae aegypti genome [3] versus $16 \%$ in the malaria mosquito genome [2]. There are also differences in the karyotype structure between the two species. The mitotic chromosome complement of Ae. aegypti consists of three pairs of metacentric chromosomes [4]. The smallest, largest and intermediate chromosomes are numbered as 1 , 2 and 3, respectively [5]. There are no sex chromosomes in Ae. aegypti; sex determination alleles have been linked to the smallest homomorphic autosome 1 [6]. By contrast, An. gambiae has two submetacentric autosomes and clearly 
distinguishable sex chromosomes: an acrocentric, halfheterochromatic X and a completely heterochromatic Y [7]. The An. gambiae genome is subdivided into two compartments - gene-rich euchromatin and gene-poor pericentromeric and intercalary heterochromatin [8]. TEs and satellites have been found to be the most abundant in the heterochromatic regions. Unlike in An. gambiae, TEs in the Ae. aegypti genome predominantly infiltrated introns of most of the protein-coding genes [1]. Although Cbanding studies located heterochromatin in centromeres of all chromosomes and in the intercalary region on the $\mathrm{q}$ arm of the homomorphic sex-determining chromosome 1 of Ae. aegypti [9-11], molecular characteristics of the heterochromatic regions in this species remain unclear.

The availability of genome sequences for mosquitoes provides an opportunity to study the molecular structures of their chromosomes and the patterns of chromosome evolution. To facilitate this investigation, physical chromosome-based maps for various species of mosquitoes have been developed [12-15]. The most detailed polytene chromosome-based physical map was constructed for the malaria vector $A n$. gambiae $[2,15,16]$. This map includes 2,000 bacterial artificial chromosome (BAC) clone markers and anchors $88 \%$ of the genome to the chromosomes. Lower resolution physical maps were also created for An. funestus [12] and An. stephensi [13]. Only about $31 \%$ of the Ae. aegypti genome was originally assigned to the chromosomes without specifying order and orientation [1] based on previous genetic mapping data [17,18]. A recent genetic mapping effort assigned $60 \%$ of the genome to 62 chromosome positions [19]. Although the physical maps of the mosquitoes are incomplete, they provide important insights into chromosomal evolution. A comparative study between $A n$. gambiae and two other malaria mosquitoes, An. funestus and An. stephensi, demonstrated that chromosome arms have different rates of evolution associated with arm-specific genomic features [20,21]. The highest rate of evolution was detected in the sex chromosome $\mathrm{X}$ in association with an abundance of TEs and tandem repeats. A comparative cytogenomic study between Aedes and Anopheles shed light on the pattern of chromosomal evolution in mosquito lineages that diverged about 145 to 200 million years ago [22] and provided some clues about the evolution of homomorphic and heteromorphic sex chromosomes [1]. Coarse-scale chromosome comparison between Ae. aegypti and An. gambiae detected whole chromosome arm translocations between chromosomes 2 and 3 as well as the conserved gene orthology between the homomorphic sex-determining chromosome 1 of $A e$. aegypti and both the $\mathrm{X}$ chromosome and autosome $2 \mathrm{R}$ arm of $A n$. gambiae [1,23]. However, a fine-scale analysis of the chromosomal rearrangements in association with the genomic landscape has not been performed. The major limitation for this analysis is a lack of sufficient information about the position of genomic supercontigs on chromosomes of Ae. aegypti.

Physical mapping on polytene chromosomes of Ae. aegypti is difficult due to the low levels of polyteny, which result in poor quality chromosome preparations [24-26]. Furthermore, fluorescent in situ hybridization (FISH) experiments are complicated by the abundance of repetitive elements, which requires using unlabeled repetitive DNA fractions to block unspecific hybridization. The first physical map for Ae. aegypti was developed by FISH of 37 markers on mitotic chromosomes from the ATC-10 cell line [27]. This map was later integrated with the genetic linkage map [17] by direct placement of 27 DNA probes containing previously mapped genetic markers to chromosomes [28]. The map was distance-based, meaning that positions of the markers were determined by direct measurements of their locations on the chromosomes from the $\mathrm{p}$ terminus (FLpter). Recently, we introduced a bandbased approach for the physical mapping of the Ae. aegypti genome to mitotic chromosomes [14,29]. Instead of previously used cell lines, which usually accumulate chromosome rearrangements [27,30], our method utilizes chromosomes from imaginal discs of fourth instar larvae. The positions of the probes are determined based on idiograms - schematic representations of the chromosome banding patterns. Idiograms have been constructed for chromosomes at early metaphase stained by YOYO-1 iodide. The three chromosomes of Ae aegypti are subdivided into 23 regions and 94 subdivisions. Using FISH, 100 BAC clones were assigned to the specific bands on idiograms. These BAC clones contained previously mapped genetic markers as determined by PCR [31]. All BAC clones were additionally ordered within each band by multicolor FISH [14]. In addition to 100 genetic markers and $183 \mathrm{Mb}$ of genomic sequences, a marker linked with sex determination [18] and 12 quantitative trait loci (QTL) associated with pathogen transmission [32-36] were also anchored to the chromosomes. However, the available physical map covered only $13.3 \%$ of the genome, and it required further improvement.

In this study, we constructed a more detailed physical map of the Ae aegypti genome. Together with our previous mapping [14], a total of $624 \mathrm{Mb}$ equal to approximately $45 \%$ of the Ae. aegypti genome were assigned to chromosome bands on idiograms. Our study revealed differences among chromosome arms in the composition of genomic features, such as genes, tandem repeats and TEs. We demonstrated that the sex-determining chromosome 1 has a significantly higher coverage of minisatellites than observed with chromosomes 2 and 3 . We also investigated chromosome rearrangements between Ae. aegypti and An. gambiae. Our data demonstrated that previously proposed whole-arm synteny $[1,23]$ is not fully preserved; a number of pericentric inversions have occurred between culicines 
and anophelines. Mapping 1:1 orthologs and microsynteny blocks common to Ae. aegypti and An. gambiae suggests a higher rate of gene reshuffling in the sex-determining chromosome of Ae. aegypti compared with chromosomes 2 and 3. Further development of a high-resolution physical map of the Ae. aegypti genome will lead to a significant improvement in the genome assembly and will guide future efforts to study genome organization and chromosome evolution in mosquitoes.

\section{Results}

\section{A physical map of the Ae. aegypti genome}

Our study developed a physical map of $45 \%$ of the $A e$. aegypti genome using FISH with mitotic chromosomes from imaginal discs of fourth instar larvae (Figure 1). The physical mapping was conducted based on previously developed idiograms for early metaphase chromosomes stained with YOYO-1 iodide [14]. For better accuracy and efficiency of mapping, we used a molecular landmarkguided mapping approach, which has been previously introduced [37]. Two-color hybridization of BAC clones was performed in the presence of three landmark probes with known locations in each of the three chromosomes of $A e$. aegypti (Figure 1). For landmarks, we utilized BAC clones that produced strong unique signals in telomeric regions of the chromosomes. Using this approach, we hybridized 400 BAC clones from the Ae. aegypti NDL BAC library [31] to the chromosomes. The positions of the BAC clones within the largest genomic supercontigs were determined by a PCR-based library screening approach. From the 400 BAC clones isolated, 368 clones were successfully hybridized and mapped to the chromosomes. Together with the previously mapped 100 genomic supercontigs [14], a total of 294 supercontigs or $624 \mathrm{Mb}$ of the Ae. aegypti genome were assigned to 91 of 94 total bands on chromosomes (Figure 2, Table 1, Additional file 1: Table S1). None of the BAC clones hybridized to region $1 \mathrm{p} 24$, pericentromeric region $1 \mathrm{p} 11$ and $3 \mathrm{q} 43$, region next to the telomere. Almost half of all supercontigs were mapped to the biggest chromosome 2 . About $45 \%$ of Ae. aegypti genomic sequences and $47 \%(7,300$ out of 15,500$)$ of the protein-coding genes were placed in the specific locations of the chromosomes. The genomic supercontigs were assigned to the chromosome bands without ordering and orientation within the band. The order of the genomic supercontigs on our map was highly consistent with a recently published genetic linkage map [19], with Spearman's rank correlation coefficients equal to $0.77,0.83$ and $0.65(P<0.05)$ for chromosomes 1,2 and 3, respectively (Additional file 2: Figure S1).

Our physical mapping detected 29 cases of potential misassembly of genomic supercontigs. In these cases, two or more BAC clones identified in the same supercontig hybridized to very different chromosomal locations. Of these, 12 supercontigs $(1.2,1.30,1.54,1.74,1.80,1.91,1.99,1.154$, $1.243,1.286,1.288$ and 1.302 ) contained only two BAC clones hybridized to the different chromosome bands, and we indicated their positions on the chromosomes as unknown (Additional file 1: Table S1). Supercontigs 1.11, $1.12,1.20,1.31$ and 1.48, which contained more than two mapped BAC clones, were assigned to the chromosomes based on the majorities of FISH results. The other 12 supercontigs $(1.1,1.3,1.7,1.25,1.44,1.50,1.76,1.98,1.148$, $1.206,1.209$ and 1.328 ) were previously assigned to chromosomes [14], and we also kept them on the map because their chromosome positions have been confirmed by genetic mapping [18]. These supercontigs are indicated in bold on the chromosome map (Figure 2). In 84 of 119 cases $(73 \%)$, when we mapped two or more BAC clones
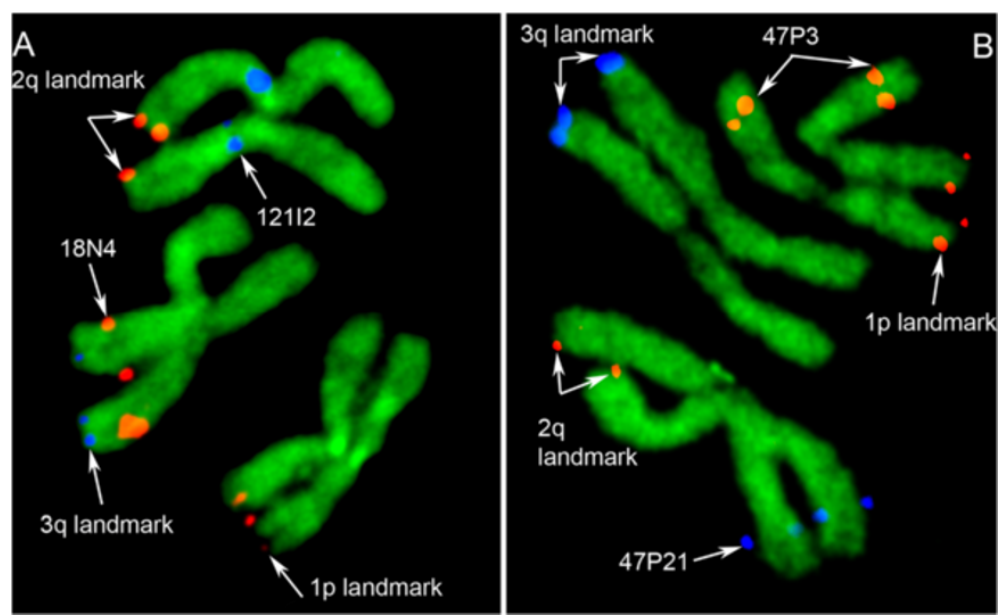

Figure 1 A landmark-guided fluorescent in situ hybridization mapping of Ae. aegypti chromosomes. The positions of bacterial artificial chromosome (BAC) clones used as chromosome arm landmarks and locations of BAC clones of interest on chromosomes (A) 2 and 3 and (B) 1 and 2 are indicated by arrows. 


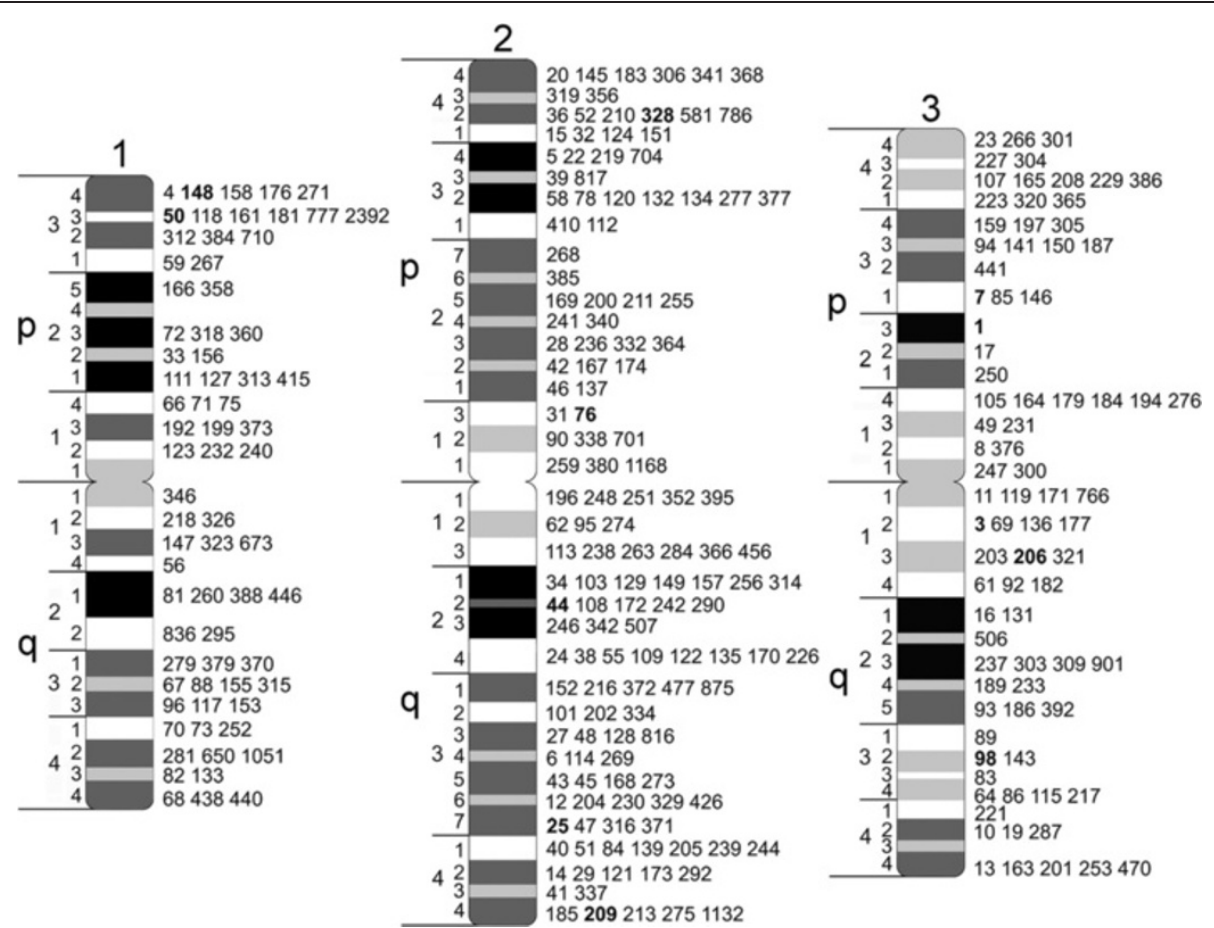

Figure 2 A physical map of the Ae. aegypti genome. Chromosome divisions and subdivisions are indicated on the left side of the idiograms. The positions of supercontigs are shown on the right side of the idiograms. Supercontig identities are indicated by the last two to four digits. Supercontigs in conflict with previous genetic mapping/genome assemblies are in bold.

from the same supercontig to the chromosomes, the mapping results were consistent with the genome data, indicating a proper assembly of these supercontigs.

\section{Genomic composition of Ae. aegypti chromosomes}

We used the physical map developed by this study to compare genomic features, such as genes, satellites and TEs, in individual chromosomes of Ae. aegypti (Figure 3). The average protein-coding gene densities per $\mathrm{Mb}$ were 10.9 in chromosome 1, 12.3 in chromosome 2 and 13.4 in chromosome 3. Although statistical analysis of proteincoding gene numbers revealed no significant differences among the chromosomes $(P=0.12)$, the sex-determining chromosome 1 was slightly but significantly different from

Table 1 Results of a physical mapping of the Ae. aegypti genome

\begin{tabular}{ll}
\hline Mapping parameters & Number (percentage) \\
\hline Number of BAC clone & 500 \\
FISH results suitable for mapping & $468(94 \%)$ \\
Assigned supercontigs & 294 \\
Assigned to chromosome 1 & $70(23 \%)$ \\
Assigned to chromosome 2 & $142(48 \%)$ \\
Assigned to chromosome 3 & $82(29 \%)$ \\
Mapped portion of the genome & $624 \mathrm{Mb}(45 \%)$ \\
\hline
\end{tabular}

BAC, bacterial artificial chromosome; FISH, fluorescent in situ hybridization. chromosome 3 , if mild criterion for pair comparisons (least significant difference test $P=0.04$ ) was used. Among chromosome arms, the lowest gene density of 9.42 per $\mathrm{Mb}$ was found in the $\mathrm{q}$ arm of the homomorphic sexdetermining chromosome (Figure 3A). Non-conservative pair comparison showed that the gene densities in chromosome $1 \mathrm{q}$ arm were significantly different from gene densities in $2 \mathrm{p}(P=0.048)$ and $3 \mathrm{q}(P=0.004)$. In contrast to genes, tandem repeats were significantly more abundant in chromosome 1 . Chromosome 1 had $6.73 \%$ of tandem repeats compared with $4.46 \%$ in chromosomes $2\left(P<10^{-7}\right)$ and $4.55 \%$ in chromosome $3\left(P<10^{-7}\right)$ (Figure 3$)$. The abundance of minisatellites (7 to 99 base-pair (bp)-long repeat units) in chromosome 1 provided the major contribution to these differences (Figure 3B). Coverage of microsatellites (2 to 6 bp-long repeat units) and large satellites (>100 bp-long repeat units) was not significantly different among the chromosomes. Our analysis also revealed significantly higher overall density of all satellites on the $\mathrm{q}$ arm (Figure 3A) of chromosome $1\left(8.80 \%, P<10^{-7}\right)$.

The overall coverage of TEs was almost equal among chromosomes: $51.9 \%$ on chromosome $1,51.8 \%$ on chromosome 2 and $53.0 \%$ on chromosome $3(P=0.25)$ (Figure 3). The analysis of various types of TEs revealed differences in classes distributed among the chromosomes (Figure 3C). For example, chromosome 1 had a lower density of Class II, DNA-mediated transposons 


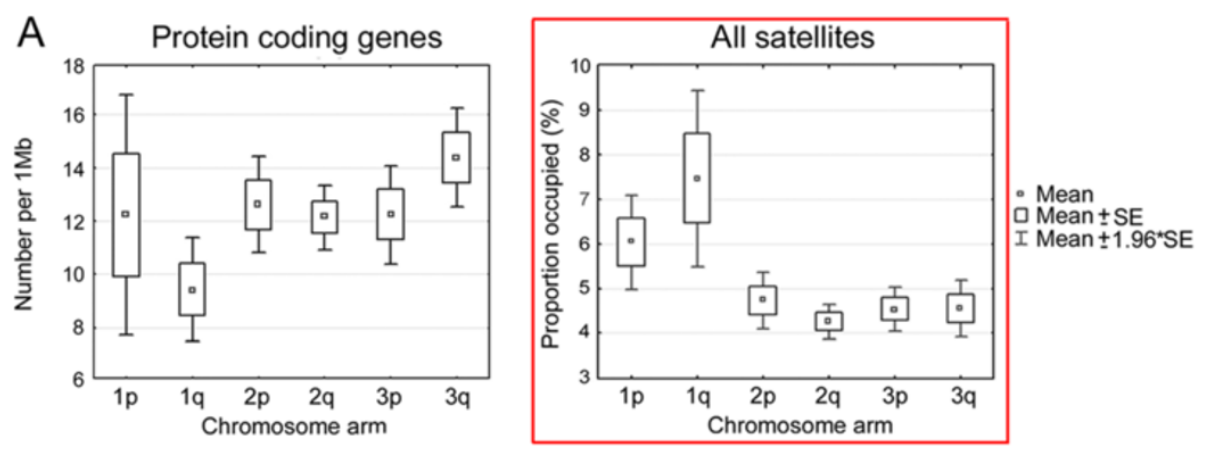

All transposable elements
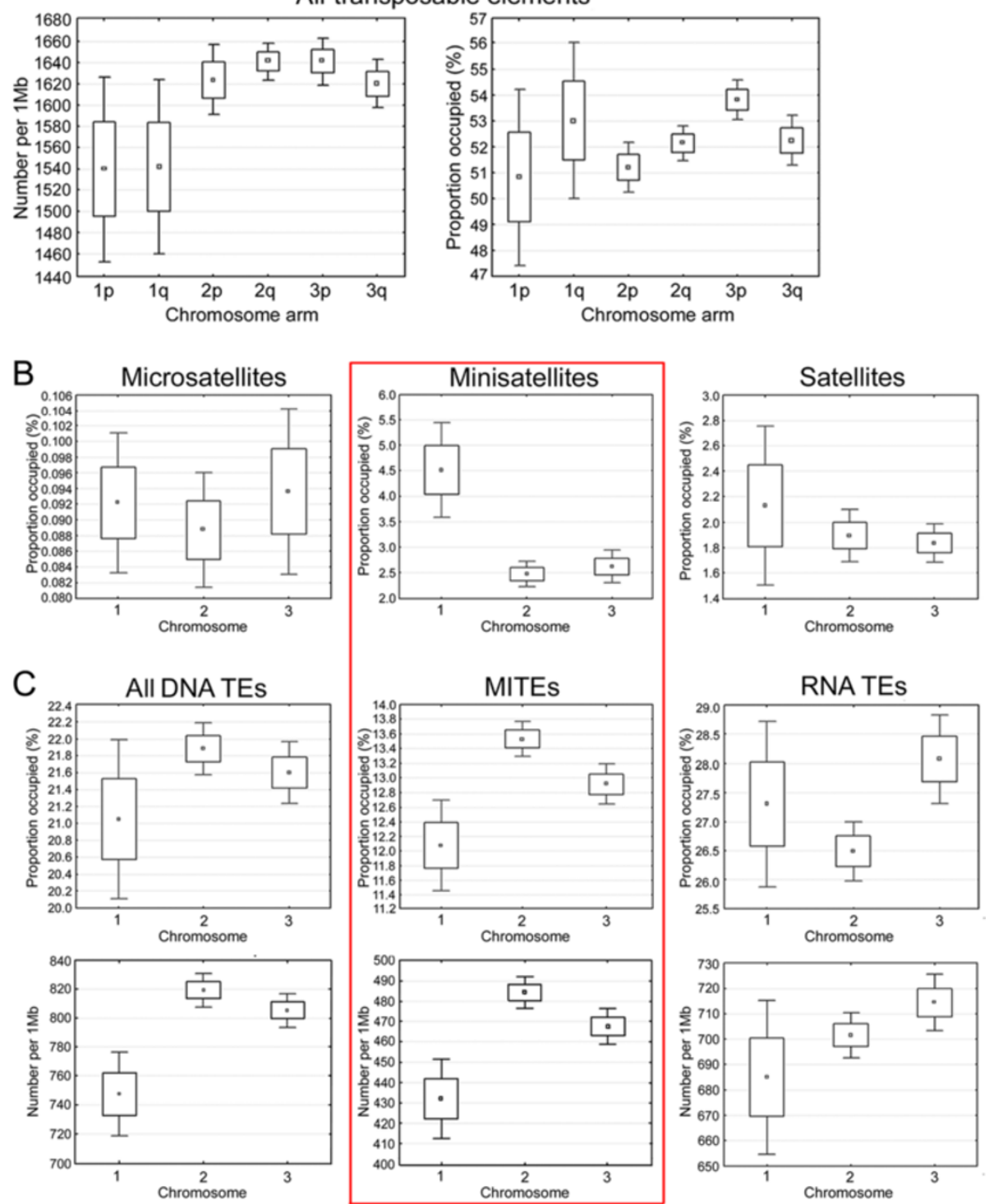

Figure 3 Distribution of molecular features in Ae. aegypti chromosome. (A) Genes, satellites and TEs in chromosome arms; (B) satellites and transposable elements in chromosomes; $(\mathbf{C})$ transposable elements in chromosomes. Densities or coverage of the molecular features per supercontig are indicated on the $\mathrm{Y}$ axis; chromosome arms or chromosomes are shown on the $\mathrm{X}$ axis. The diagrams with the most striking differences in molecular features between chromosome and chromosome arms are in red boxes. Mb: mega base pairs; MITEs: miniature inverted-repeat transposable element; SE: standard error; TEs: transposable elements. 
(747.2 per $\mathrm{Mb}$ ) than chromosomes 2 (819.1 per $\mathrm{Mb}$, $\left.P<10^{-7}\right)$ and $3\left(805.1\right.$ per $\left.\mathrm{Mb}, P=1.3 \times 10^{-4}\right)$. All chromosomes differed in the abundance (coverage and counts) of miniature inverted-repeat TEs (MITEs). This class of short, 500 bp-long TEs belongs to DNA-mediated TEs. MITEs were most abundant in chromosome $2(13.5 \%, 484.3$ per $\mathrm{Mb})$ as compared with chromosomes $1\left(P<10^{-4}\right)$ and 3 $\left(P=1.3 \times 10^{-2}\right)$. Chromosome 3 had a higher coverage of Class I, RNA-mediated TEs than chromosome 2 (26.5\% versus $28.1 \%, P=2 \times 10^{-2}$ ). Interestingly, the overall densities of TEs were slightly lower in sex-determining chromosome 1 (1,540 per Mb) than in chromosomes $2(1,634$ per $\left.\mathrm{Mb} ; P=2.2 \times 10^{-5}\right)$ and $3\left(1,630\right.$ per $\left.\mathrm{Mb} ; P=2.73 \times 10^{-4}\right)$. This fact can be explained by the differences in sizes between different classes of TEs and preferable location of the TEs in gene introns that makes them abundant in the gene-rich environment on Ae. aegypti chromosomes 2 and 3 .

In addition to the interchromosome comparison, we also analyzed the landscape of molecular features along the three chromosomes of Ae. aegypti (Figure 4). Despite the fact that the physical map constructed here covers only approximately $45 \%$ of the Ae. aegypti genome, our study demonstrated that the densities of genes in areas around the centromeres of chromosomes 1 and 2 were lower than the average in the chromosomes. By contrast, the coverage of satellites was significantly higher around the centromeres in regions 1q11, 2p11 and 3p11 $\left(P<10^{-5}\right)$. A dearth of genes (5.9 per $\mathrm{Mb}, P<10^{-5}$ ) and abundance of satellites $\left(11.54 \%, P<10^{-5}\right)$ were also found in region $1 \mathrm{q} 22$. The location of the ribosomal genes in this region has been shown previously [29]. Moreover, scaffold 1.836, which contains the rDNA region, had even higher satellite content $(24.1 \%)$, whereas the average satellite content in all chromosomes was equal to $5.02 \%$. Thus, pericentromeric areas and region 1q22 have molecular characteristics of heterochromatin [38]. An additional peak of satellites was found in the subtelomeric region 1q42 to $1 \mathrm{q} 43$ (17.97\% and $12.96 \%)$. TEs did not demonstrate significant differences in their distribution along the chromosomes.

\section{Chromosome rearrangements between Ae. aegypti and An. gambiae}

The synteny between the chromosome regions in Ae. aegypti and An. gambiae was investigated using the physical map developed in this study. This investigation of synteny excluded 40 misassembled supercontigs identified by genetic linkage mapping [19]. A total of 2,335 out of 5,265 Ae. aegypti-An. gambiae orthologs were mapped to the 254 properly assembled supercontigs of the Ae. aegypti genome (Figure 5). Almost each subregion of Ae. aegypti had orthologs from more than one An. gambiae chromosome. Nine from 94 subregions contained zero orthologs due to the low number of mapped supercontigs. The number of orthologs per region varied from 1 to 75 . The number of shared orthologs between Ae. aegypti and An. gambiae in each region is shown by the color intensities in Figure 5. A higher number of shared orthologs per region were found in the more densely mapped chromosome 2. In general, the results recapitulated the Ae. aegypti and An. gambiae chromosome arm synteny identified previously $[1,23]$. However, the physical map developed here provides a more detailed view of synteny at subregion resolution. Our data support the previous finding that the $1 \mathrm{p}$ and 1q arms of Ae. aegypti mostly contain the genomic material from the $\mathrm{X}$ and $2 \mathrm{R}$ chromosome arms of $A n$. gambiae, respectively, supporting the idea of large translocation between the $\mathrm{X}$ and part of the $2 \mathrm{R}$ chromosome arm. In addition, we found a clear correspondence between the $\mathrm{X}$ chromosome of $A n$. gambiae and region 1q22, known to contain the ribosomal locus in Ae. aegypti. We also found a synteny block between the $2 \mathrm{R}$ arm of $A n$. gambiae and the 1p arm of Ae. aegypti in subregion $1 \mathrm{p} 12$. These results indicate the possibility of pericentric inversions during the evolution of the sex-determining chromosome in mosquitoes. The presence of additional large blocks from $A n$. gambiae arms $2 \mathrm{~L}$ and $3 \mathrm{R}$ on chromosome 1 of Ae. aegypti suggests even more complex evolution of this chromosome in Ae. aegypti. Observations of the synteny between chromosomes 2 and 3 in Ae. aegypti and An. gambiae clearly aligned with the previous data supporting whole-arm translocations $[1,23]$. However, the data also suggest the possibility of exchanges in genetic material by pericentric inversions between arms in the evolution of Ae. aegypti chromosomes 2 and 3.

In addition to the insights regarding the evolution of chromosomes between Ae. aegypti and An. gambiae, we attempted to rank the amount of gene order reshuffling within the chromosome arms (Figure 6). The gene order on the newly developed physical map of Ae. aegypti was compared to the gene order in the genome map of An. gambiae [2,16]. The genes of the Ae. aegypti and An. gambiae chromosomes were mapped based on 1:1 orthologs (Figure 6A) and microsynteny blocks (Figure 6B) at subregion resolution. A microsynteny block is defined as the sharing of nine or more orthologs between subregions of Ae. aegypti and An. gambiae. Table 2 shows the percentage of orthologs found in microsynteny blocks for each chromosome: chromosome arms 1p (15\%), 1q (11\%) and $3 p(34 \%)$ had the lowest percentages. In agreement with previous observation of a higher rate of evolution of the $2 \mathrm{R}$ arm in Anopheles [21], these data support the idea that the sex-determining chromosome 1 and arm $3 p$ in Ae. aegypti had the highest rate of gene order reshuffling. In particular, arm $3 \mathrm{p}$ in Ae. aegypti, homologous to arm 2R in An. gambiae, demonstrated the highest rate of rearrangements among the autosomes of that species. Arm 2q 


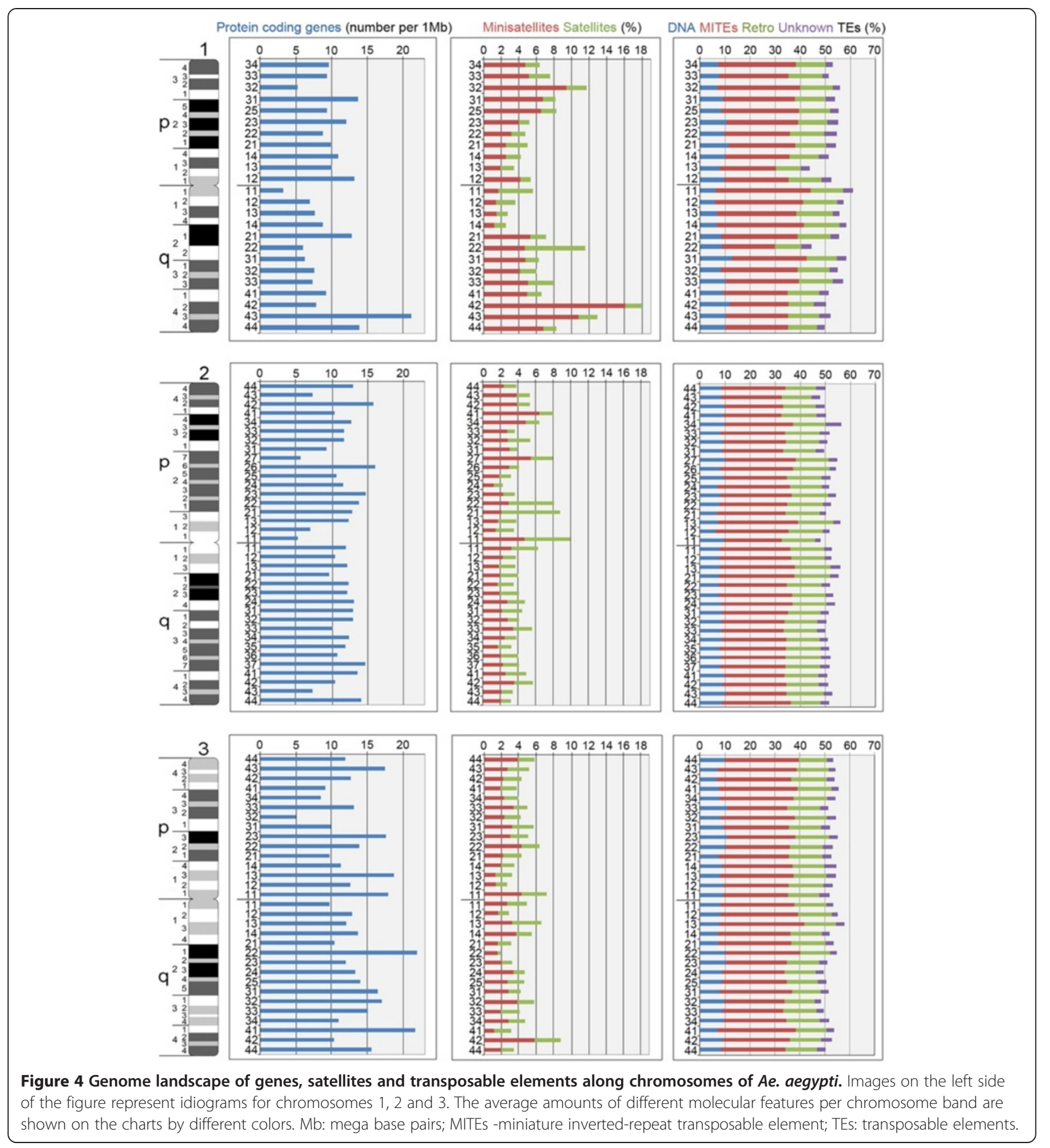

in Ae. aegypti and arm 3R in An. gambiae shared the greatest number of orthologs (602), microsynteny blocks (24) and percentage of orthologs within microsynteny blocks (55\%), indicating that this arm had the highest degree of conservation. Although we did not attempt to normalize the data for the gene densities and number of supercontigs hybridized in each subregion, these results were still indicative of the conserved regions of the Ae. aegypti and An. gambiae genomes.

\section{Discussion}

Availability of previously constructed physical maps for An. gambiae $[2,15,16]$ and the newly developed physical map for Ae. aegypti allows comparison of the genomic 


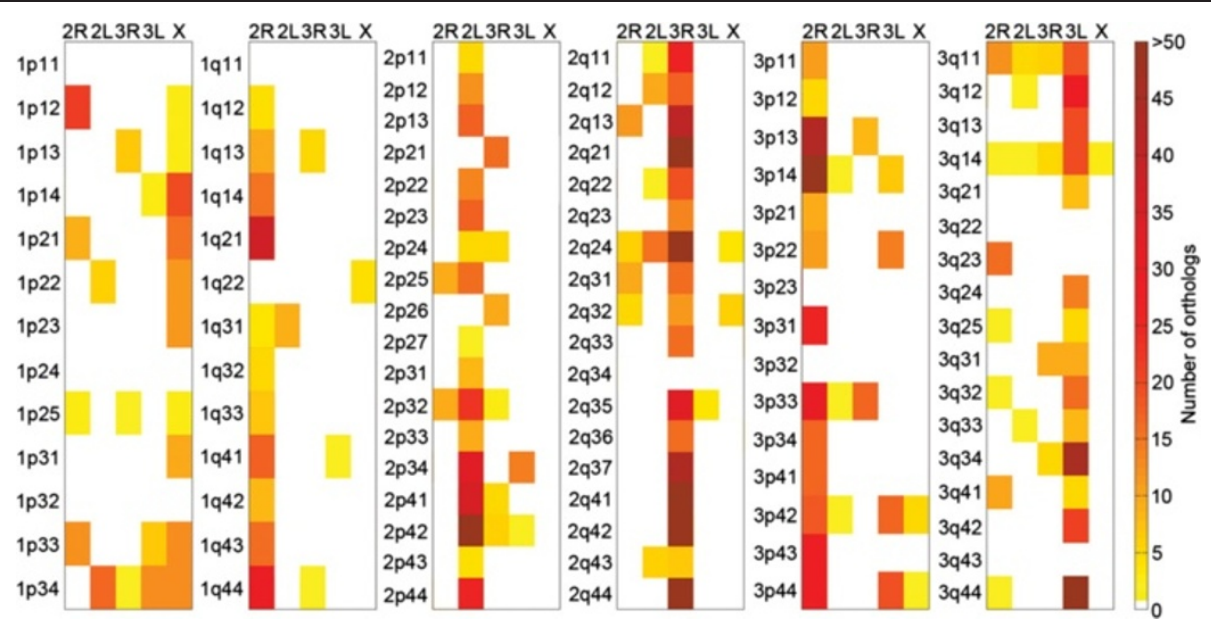

Figure 5 Chromosome map of orthologous genes between Ae. aegypti and An. gambiae. The intensities of the color indicate differences in numbers of orthologs in each region (scale in the right column). The $Y$ axis shows subdivisions of the Ae. aegypti chromosomes. The $X$ axis represents chromosome arms of An. gambiae.

composition of the chromosomes in two species of mosquitoes. Cytogenetic studies conducted in the past demonstrated striking differences in chromosome organization between the two mosquitoes. Despite the fact that both mosquitoes have chromosome number $2 \mathrm{n}$ equal to six, the length of the chromosomes and the karyotype structure are different [4]. The average length of the $\mathrm{X}$ chromosome and two autosomes together in malaria mosquitoes is equal to about $11 \mu \mathrm{m}$ [39]. Malaria mosquitoes have clearly distinguishable heteromorphic sex chromosomes $\mathrm{X}$ and $\mathrm{Y}$. These two chromosomes contain large amounts of heterochromatin. Almost half of the $\mathrm{X}$ chromosome is represented by heterochromatic blocks [7]. The Y chromosome is almost entirely heterochromatic. Autosomes 2 and 3 also contain large heterochromatic blocks around the centromeres. The analysis of the polytene chromosomes found three regions of intercalary heterochromatin in arms $2 \mathrm{~L}, 3 \mathrm{R}$ and $3 \mathrm{~L}$ in addition to pericentromeric heterochromatin [8]. Chromosomes of Ae. aegypti are approximately 2.3 times longer than chromosomes of $A n$. gambiae. The average lengths of the chromosomes at metaphase are equal to $7.15 \mu \mathrm{m}$ for chromosome 1, 9.46 $\mu \mathrm{m}$ for chromosome 2 and $8.36 \mu \mathrm{m}$ for chromosome 3 [29]. Sex in Ae aegypti is determined by a locus on the smallest homomorphic autosome 1 [6]. The position of heterochromatin in Ae. aegypti chromosomes was demonstrated using a Giemsa C-banding technique $[10,40]$. Cbands were originally found in pericentromeric regions of female sex-determining chromosome 1 and autosomes 2 and 3. An additional intercalary C-band was found in the female sex-determining chromosome 1 . Male sexdetermining chromosome 1 demonstrated no heterochromatin in two original reports. However, the presence of heterochromatin in the pericentromeric region of the male-determining chromosome has been demonstrated by a silver-staining technique [9]. Comparative studies of different strains of Ae. aegypti [11,40] and natural populations in Brazil [41] demonstrated polymorphism in the presence/absence and sizes of intercalary band in both male- and female-determining chromosomes 1 . An additional polymorphic intercalary C-band was also found in chromosome 3 in a Brazilian population of $A e$. aegypti [41].

The composition of genes, satellites and TEs in chromosomes of Ae. aegypti and An. gambiae is compared in Figure 7A. Gene densities were lower in sex-determining chromosome 1 in Ae. aegypti and in the $\mathrm{X}$ chromosome of An. gambiae. Satellites displayed an alternative pattern and were more abundant in the sex-determining chromosomes 1 of Ae. aegypti and $\mathrm{X}$ chromosome of An. gambiae. The distributions of genes and satellites along the chromosomes of Ae. aegypti (Figure 4) in general followed the C-banding patterns $[10,40]$. We found significantly lower density of genes and higher coverage of repeats in centromeres and in the ribosomal locus 1q22, which forms an additional C-band on the q arm of chromosome 1 . The polymorphism of this band between strains and populations of Ae aegypti, which has previously been demonstrated $[11,40,41]$, can be explained by the differences in copy numbers of ribosomal genes. Interestingly, the anchor marker for the sex determination locus was mapped in the neighboring region 1q21 represented by a large condensed band on YOYO-1stained chromosomes [14]. Nevertheless, region 1q21 had lower repeat coverage and contained a significantly higher density of genes. Similar to D. melanogaster [38,42], the majority of the TEs in the malaria mosquito genome were distributed in heterochromatic areas around the 


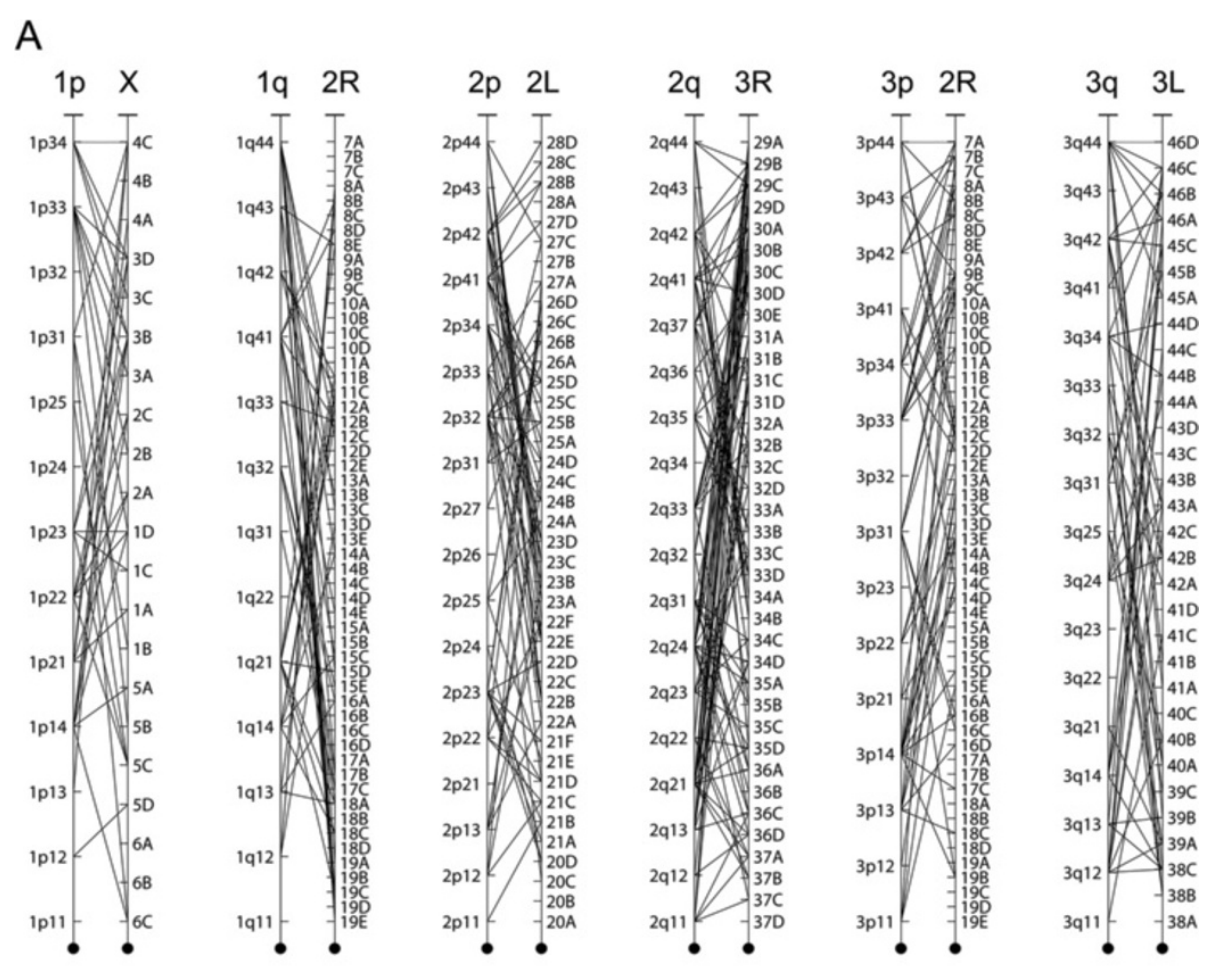

B
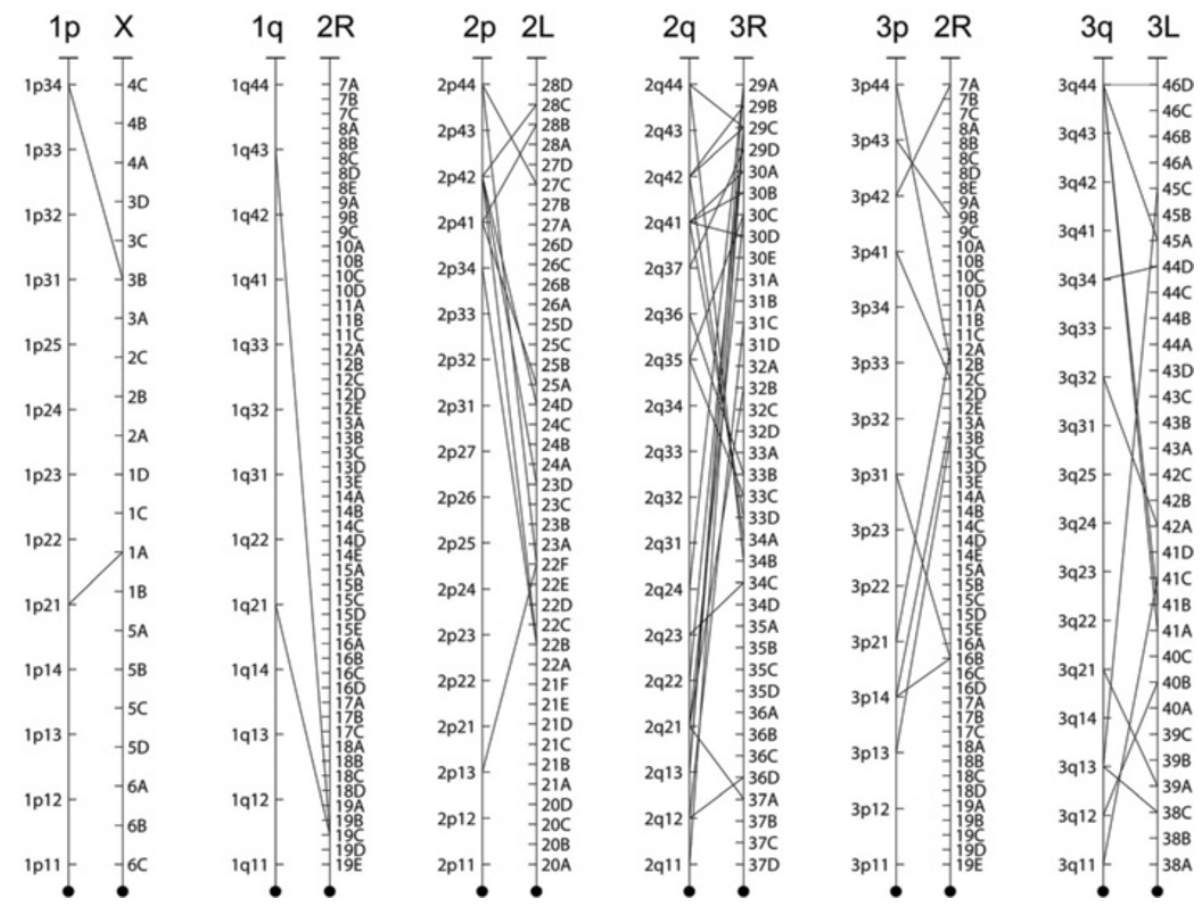

Figure 6 Shuffling gene order in chromosomes of Ae. aegypti and An. gambiae. Correspondence of (A) 1:1 orthologs and (B) microsynteny blocks are shown by lines.

centromeres [8]. The TE coverage in pericentromeric heterochromatin of An. gambiae reached 53\%. Surprisingly, TEs in Ae. aegypti do not follow the pattern in An. gambiae. By contrast, TEs in Ae. aegypti chromosomes are mostly spread in euchromatic regions because of their tendency to localize in gene introns [1]. According to our current estimate, the mapped portion of the Ae. aegypti genome contains about 5\% tandem repeats and 52\% TEs. 
Table 2 Orthologs and microsynteny blocks in the Ae. aegypti and An. gambiae genome maps

\begin{tabular}{|c|c|c|c|c|c|c|c|}
\hline Chromosome arms (Aa/Ag) & $1 \mathrm{p} / \mathrm{X}$ & $1 q / 2 R$ & $2 p / 2 L$ & $2 q / 3 R$ & $3 p / 2 R$ & $3 q / 3 L$ & total \\
\hline Orthologs in synteny blocks & 18 & 18 & 127 & 332 & 104 & 151 & 750 \\
\hline Total orthologs & 120 & 159 & 332 & 602 & 308 & 319 & 1840 \\
\hline Percent in blocks & $15 \%$ & $11 \%$ & $38 \%$ & $55 \%$ & $34 \%$ & $47 \%$ & $41 \%$ \\
\hline
\end{tabular}

Aa, Ae. aegypti; Ag, An. gambiae.

Thus, the euchromatic regions of Ae. aegypti chromosomes have the same coverage of TEs as heterochromatin of An. gambiae.

The 2R arm of An. gambiae [1] has the highest gene densities and the lowest coverage of tandem repeats and densities of TEs [21]. Interestingly, chromosome arm 1q, which is homologous to the $2 \mathrm{R}$ arm of An. gambiae, has by contrast the lowest gene densities and the highest coverage of tandem repeats in Ae. aegypti (Figure 7). However, the $\mathrm{q}$ arm of chromosome 1 cannot be considered entirely heterochromatic as was previously suggested [28]. Even with approximately $45 \%$ of the genome placement to the chromosomes, we found that at least 594 (8\%) of genes were located in this arm. The heterochromatic nature of this particular arm can be explained by the presence of the ribosomal DNA locus and the sex determination locus that can directly stimulate accumulation of the repetitive DNA. Formation of the heterochromatin around the ribosomal locus prolongs lifespan by silencing transcription of the ribosomal genes in Drosophila [43]. Another study conducted on Drosophila showed that heterochromatin controls male viability by regulating genes in the sex determination pathway [44]. Thus, our data suggest that a high coverage of satellites rather than TEs could be the major characteristic of the heterochromatin in Ae. aegypti. We also argue that the genomic composition of the sexdetermining chromosome 1 in Ae. aegypti is influenced by the presence of the sex-determining locus and ribosomal genes.

Our analysis of the chromosome rearrangements revealed that the pattern of chromosome evolution between An. gambiae and Ae. aegypti was more complex than previously suggested [1]. In addition to the known exchange of genomic material between chromosome $\mathrm{X}$ and a part of the 2R arm of An. gambiae in chromosome

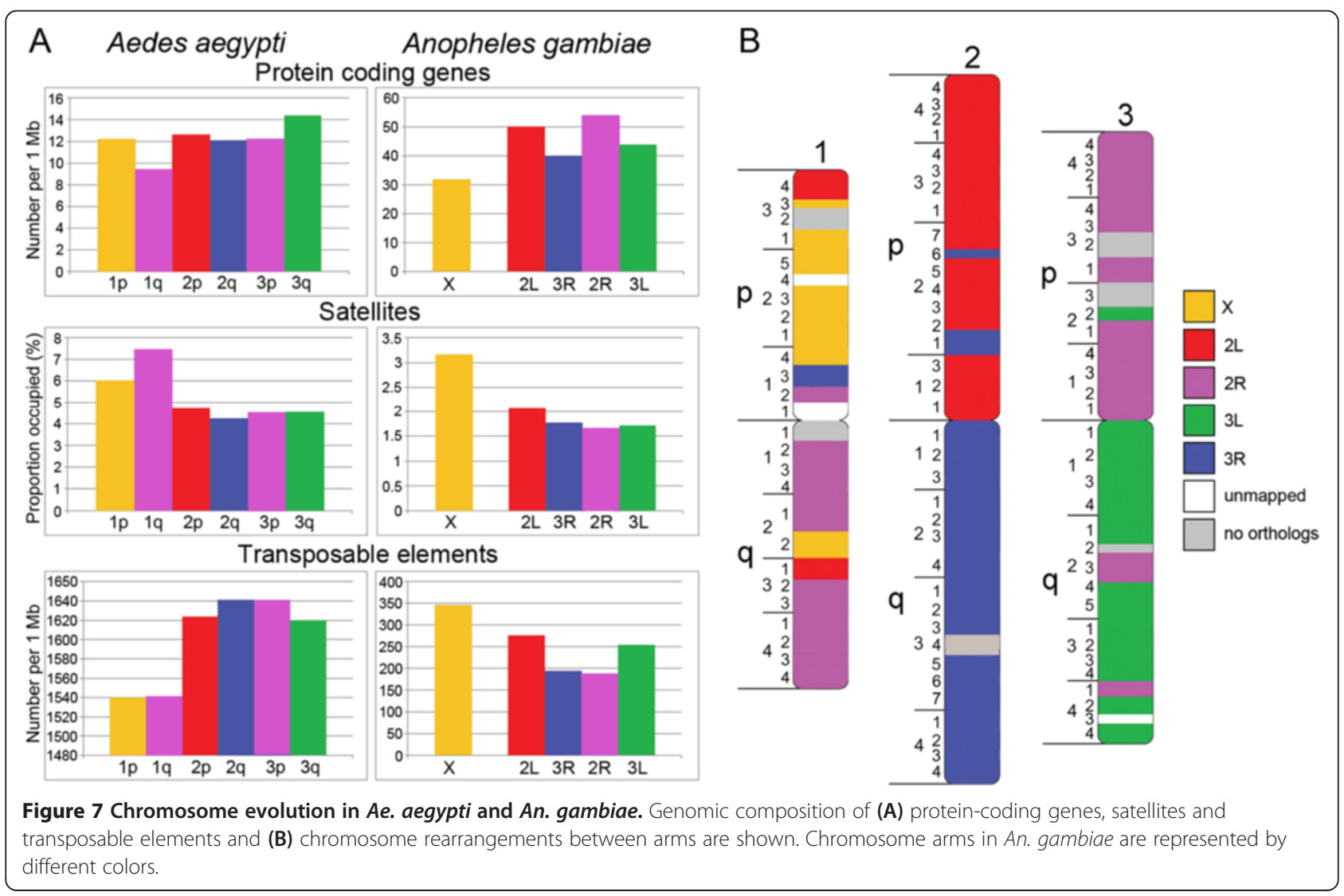


1 of Ae. aegypti, a number of pericentric inversions have reshuffled the genetic materials between chromosome arms 1p and 1q (Figure 7B). Similar patterns of pericentric inversions were also found in autosomes 2 and 3 as an addition to the previously determined whole-arm translocation [1]. Our study has also shown that gene order within chromosome arms of Ae aegypti was poorly conserved, because of multiple paracentric inversion. Similar types of chromosome rearrangements have been determined in Drosophila [45]. For example, the metacentric X chromosomes in D. willistoni, D. pseudoobscura and D. persimilis were generated by a fusion of the $\mathrm{X}$ and autosomal 3L arm of D. melanogaster. Additional pericentric inversions were detected in D. erecta, D. yakuba, $D$. pseudoobscura and D. persimilis compared with the chromosome pattern of D. melanogaster. Similarly to the heteromorphic sex chromosomes in Anopheles [21] and Drosophila [46], homomorphic sex-determining chromosomes 1 in Ae. aegypti demonstrated the highest rate of the rearrangements among the chromosomes. However, unlike in Drosophila and Anopheles, chromosome 1 in Ae. aegypti was not enriched with TEs, which can be associated with inversion formation [47-50]. Instead, we demonstrated a high coverage of minisatellites suggesting that tandem repeats might play a special role in chromosome evolution of Aedes mosquitoes. Indeed, simple repeats can be involved in DNA breaks and chromosome rearrangements by the formation of hairpin and cruciform structures [51]. Thus, despite the morphological and molecular differences between homomorphic sex-determining chromosomes in Aedes and heteromorphic sex chromosomes in Anopheles, they both display the rapid rate of gene order evolution.

\section{Conclusions}

This study developed a physical map comprising $45 \%$ of the yellow fever mosquito genome by assigning $624 \mathrm{Mb}$ of 294 genomic supercontigs to chromosome bands. This map guided the analyses of the genes, satellites and TE landscapes in the Ae. aegypti genome and provided important insights into chromosome evolution in mosquitoes. Lower gene densities and higher satellite DNA content were detected in pericentromeric regions and region 1q22 in the homomorphic sex-determining chromosome 1, which also contains the ribosomal DNA locus. These regions can be defined as heterochromatic in Ae. aegypti chromosomes. In contrast to satellites, genes and TEs were more abundant in chromosomes 2 and 3, and also in euchromatic areas of the chromosomes. A comparative genomic analysis with An. gambiae demonstrated that the whole-arm synteny was not fully preserved. Multiple pericentric inversions substantially reshuffled the genetic material of the $1 \mathrm{p}$ and $1 \mathrm{q}$ arms. Despite this reshuffling, the homologies between $1 \mathrm{p}$ and $\mathrm{X}$ chromosome, as well as between $1 \mathrm{q}$ and $2 \mathrm{R}$ arm of An. gambiae, are evident. The homomorphic sex-determining chromosome 1 demonstrated the highest rate of the chromosome rearrangements. We believe that additional physical mapping is still needed to improve the current fragmented genome assembly of Ae. aegypti. Assignment and orientation of the supercontig assemblies of the Ae. aegypti genome to the chromosomes will facilitate more advanced studies of the genome organization and chromosome evolution in mosquitoes.

\section{Methods \\ Mosquito strain}

This study was performed with the Liverpool IB-12 strain of Ae aegypti, which originated from the Liverpool strain following several rounds of inbreeding and was previously used for the genome sequencing project of Ae. aegypti [1]. The Liverpool strain has also been utilized for geneticlinkage and QTL mapping studies $[17,34,35]$.

\section{Fluorescent in situ hybridization}

Slides of mitotic chromosomes were prepared from imaginal discs of fourth instar larvae following published protocols [14,29,39]. Samples of BAC clone DNA were prepared by Clemson University Genomics Institute in 96-well plates. FISH was performed as previously described $[14,39]$. A two-color version of FISH was used for localizing BAC positions on mitotic chromosomes. BAC DNA for hybridization was labeled with Cy3- or Cy5dUTP (GE Healthcare UK Ltd., Amersham, UK) by nick translation. Chromosomes were stained with YOYO-1 iodide (Invitrogen Corporation, Grand Island, NY, USA). Slides were analyzed using a Zeiss LSM 510 Laser Scanning Microscope (Carl Zeiss Microimaging, Inc., Thornwood, NY, USA) at $600 \times$ magnification.

\section{Genomic features analysis}

For analysis of the genetic element distribution on the chromosomes, we used the sequence data from Vector Base [52] regarding each supercontig. Gene density was calculated based on the number of protein-coding genes belonging to the individual supercontigs per $\mathrm{Mb}$ using the BioMart tool of Vector Base [53]. Repetitive DNA content was analyzed using Tandem Repeat Finder with basic parameters [54]. Tandem repeats were defined by motif sizes from 2 to 6 , from 7 to 99 , and from 100 or more as microsatellites, minisatellites and satellites, respectively. Analysis of TEs was performed using Repeat Masker (version 3.2.9) [55] at the default settings using the Ae. aegypti TEs in TEfam database [56] as the custom repeat library. Repeat Masker output was then used to count the frequency of occurrence and the base occupied by each TE in each supercontig. 


\section{Chromosome synteny and ortholog mapping}

A collection of 5,265 previously identified orthologs in Ae. aegypti and An. gambiae [1] was downloaded from OrthoDB [57]. This collection was used to test for chromosome synteny between the two species. Among these 2,335 orthologs were located in correctly assembled supercontigs [19] within the physical map of Ae aegypti. Chromosome synteny was then tested for in each subregion: each Ae. aegypti subregion was identified with the $A n$. gambiae chromosomes sharing the greatest number of orthologs. Physical maps of 1:1 orthologs (at subregion resolution) were constructed using the physical map of Ae. aegypti and the genome map of An. gambiae. Microsynteny blocks, defined here as the sharing of nine or more orthologs between subregions of Ae. aegypti and An. gambiae, were also mapped.

\section{Additional files}

Additional file 1: Table S1. Supercontig and BAC clone positions on Ae. aegypti chromosomes. Major signals are indicated by asterisks. Conflict mapping data are in bold. BAC AC\#, BAC clone accession number; NA, not applicable; SC, supercontig; $\wedge$ additional signals.

Additional file 2: Figure S1. The correlation between the physical band position of a supercontig on our physical map and its $\mathrm{CM}$ position on the genetic linkage map [19]. Spearman's rank correlation coefficients equal to $0.77,0.83$ and $0.65(P<0.05)$ were determined for chromosomes (A) 1, (B) 2 and (C) 3, respectively.

\section{Abbreviations}

BAC: bacterial artificial chromosome; bp: base pairs; FISH: fluorescent in situ hybridization; Mb: mega base pairs; MITEs: miniature inverted-repeat transposable elements; PCR: polymerase chain reaction; QTL: quantitative trait loci; TE: transposable element.

\section{Competing interests}

The authors declare that they have no competing interest.

\section{Authors' contributions}

MVS, DWS and IVS designed experiments. VAT and BSdeB performed experiments. VAT, NAK, CM and ZT performed bioinformatics analysis. MVS, VAT, NAK and IVS wrote the manuscript. DWS provided resources. All authors read and approved the final manuscript.

\section{Acknowledgements}

This research was funded by grant R21 Al088035 (to MVS) from NIH/NIAID. Authors thank William C. Black for the productive discussion of the manuscript, Joanne Cunningham for the technical help and Melissa Wade for editing the manuscript.

\section{Author details}

'Department of Entomology, Fralin Life Science Institute, Virginia Tech, Blacksburg, VA, USA. ²Department of Genomics, Bioinformatics, and Computational Biology, Virginia Tech, Blacksburg, VA, USA. ${ }^{3}$ Department of Biological Sciences, Eck Institute for Global Health, University of Notre Dame, Notre Dame, IN, USA. ${ }^{4}$ Virginia Bioinformatics Institute, Virginia Tech, Blacksburg, VA, USA. ${ }^{5}$ Department of Biochemistry and Fralin Life Science Institute, Virginia Tech, Blacksburg, VA, USA.

Received: 28 March 2014 Accepted: 1 April 2014

Published: 14 April 2014

\section{References}

1. Nene V, Wortman JR, Lawson D, Haas B, Kodira C, Tu ZJ, Loftus B, Xi Z, Megy K, Grabherr M, Ren Q, Zdobnov EM, Lobo NF, Campbell KS, Brown SE, Bonaldo MF, Zhu J, Sinkins SP, Hogenkamp DG, Amedeo P, Arensburger P, Atkinson PW, Bidwell S, Biedler J, Birney E, Bruggner RV, Costas J, Coy MR, Crabtree J, Crawford M, et al: Genome sequence of Aedes aegypti, a major arbovirus vector. Science 2007, 316:1718-1723.

2. Holt RA, Subramanian GM, Halpern A, Sutton GG, Charlab R, Nusskern DR, Wincker P, Clark AG, Ribeiro JM, Wides R, Salzberg SL, Loftus B, Yandell M, Majoros WH, Rusch DB, Lai Z, Kraft CL, Abril JF, Anthouard V, Arensburger P, Atkinson PW, Baden $H$, de Berardinis V, Baldwin D, Benes V, Biedler J, Blass C, Bolanos R, Boscus D, Barnstead M, et al: The genome sequence of the malaria mosquito Anopheles gambiae. Science 2002, 298:129-149.

3. Severson DW, Behura SK: Mosquito genomics: progress and challenges. Annu Rev Entomol 2012, 57:143-166.

4. Rai KS: A comparative study of mosquito karyotypes. Ann Ent Soc Am 1963, 56:160-170.

5. McDonald PT, Rai KS: Correlation of linkage groups with chromosomes in the mosquito, Aedes aegypti. Genetics 1970, 66:475-485.

6. McClelland GAH: Sex-linkage in Aedes aegypti. Trans Roy Soc Trop Med Hyg 1962, 56:4.

7. Coluzzi M, Sabatini A: Cytogenetic observations on species A and B of the Anophles gambaie complex. Parassitologia 1967, 9:73-88.

8. Sharakhova MV, George P, Brusentsova IV, Leman SC, Bailey JA, Smith CD, Sharakhov IV: Genome mapping and characterization of the Anopheles gambiae heterochromatin. BMC Genomics 2010, 11:459.

9. Motara MA, Pathak S, Satya-Prakash KL, Hsu TC: Argentophylic structures of spermatogenesis in the yellow fever mosquito. J Hered 1985, 76:295-300.

10. Motara MA, Rai KS: Chromosomal differentiation in two species of Aedes and their hybrids revealed by Giemsa C-banding. Chromosoma 1977, 64:125-132.

11. Wallace AJ, Newton ME: Heterochromatin diversity and cyclic responses to selective silver staining in Aedes aegypti (L.). Chromosoma 1987, 95:89-93.

12. Sharakhov IV, Serazin AC, Grushko OG, Dana A, Lobo N, Hillenmeyer ME, Westerman R, Romero-Severson J, Costantini C, Sagnon N, Collins FH, Besansky NJ: Inversions and gene order shuffling in Anopheles gambiae and A. funestus. Science 2002, 298:182-185.

13. Sharakhova MV, Xia A, Tu Z, Shouche YS, Unger MF, Sharakhov IV: A physical map for an Asian malaria mosquito, Anopheles stephensi. Am J Trop Med Hyg 2010, 83:1023-1027.

14. Timoshevskiy VA, Severson DW, Debruyn BS, Black WC, Sharakhov IV, Sharakhova MV: An integrated linkage, chromosome, and genome map for the yellow fever mosquito Aedes aegypti. PLoS Negl Trop Dis 2013, 7:e2052.

15. Sharakhova MV, Hammond MP, Lobo NF, Krzywinski J, Unger MF, Hillenmeyer ME, Bruggner RV, Birney E, Collins FH: Update of the Anopheles gambiae PEST genome assembly. Genome Biol 2007, 8:R5.

16. George P, Sharakhova MV, Sharakhov IV: High-resolution cytogenetic map for the African malaria vector Anopheles gambiae. Insect Mol Biol 2010, 19:675-682.

17. Severson DW, Mori A, Zhang Y, Christensen BM: Linkage map for Aedes aegypti using restriction fragment length polymorphisms. J Hered 1993, 84:241-247.

18. Severson DW, Meece JK, Lovin DD, Saha G, Morlais I: Linkage map organization of expressed sequence tags and sequence tagged sites in the mosquito, Aedes aegypti. Insect Mol Biol 2002, 11:371-378.

19. Juneja P, Osei-Poku J, Ho YS, Ariani CV, Palmer WJ, Pain A, Jiggins FM: Assembly of the genome of the disease vector Aedes aegypti onto a genetic linkage map allows mapping of genes affecting disease transmission. PLoS Negl Trop Dis 2014, 8:e2652.

20. Sharakhova MV, Xia A, Leman SC, Sharakhov IV: Arm-specific dynamics of chromosome evolution in malaria mosquitoes. BMC Evol Biol 2011, 11:91.

21. Xia A, Sharakhova MV, Leman SC, Tu Z, Bailey JA, Smith CD, Sharakhov IV: Genome landscape and evolutionary plasticity of chromosomes in malaria mosquitoes. PLoS One 2010, 5:e10592.

22. Krzywinski J, Wilkerson RC, Besansky NJ: Toward understanding Anophelinae (Diptera, Culicidae) phylogeny: insights from nuclear single-copy genes and the weight of evidence. Syst Biol 2001, 50:540-556.

23. Severson DW, DeBruyn B, Lovin DD, Brown SE, Knudson DL, Morlais I: Comparative genome analysis of the yellow fever mosquito Aedes aegypti with Drosophila melanogaster and the malaria vector mosquito Anopheles gambiae. J Hered 2004, 95:103-113. 
24. Sharma GP, Mittal OP, Chaudhry S, Pal V: A preliminary map of the salivary gland chromosomes of Aedes (Stegomyia) aegypti (Culicadae, Diptera). Cytobios 1978, 22:169-178.

25. Campos J, Andrade CF, Recco-Pimentel SM: A technique for preparing polytene chromosomes from Aedes aegypti (Diptera, Culicinae). Mem Inst Oswaldo Cruz 2003, 98:387-390.

26. Campos J, Andrade CF, Recco-Pimentel SM: Malpighian tubule polytene chromosomes of Culex quinquefasciatus (Diptera, Culicinae). Mem Inst Oswaldo Cruz 2003, 98:383-386.

27. Brown SE, Menninger J, Difillipantonio M, Beaty BJ, Ward DC, Knudson DL: Toward a physical map of Aedes aegypti. Insect Mol Biol 1995, 4:161-167.

28. Brown SE, Severson DW, Smith LA, Knudson DL: Integration of the Aedes aegypti mosquito genetic linkage and physical maps. Genetics 2001, 157:1299-1305.

29. Sharakhova MV, Timoshevskiy VA, Yang F, Demin S, Severson DW, Sharakhov IV: Imaginal discs: a new source of chromosomes for genome mapping of the yellow fever mosquito Aedes aegypti. PLoS Negl Trop Dis 2011, 5:e1335

30. Steiniger GE, Mukherjee AB: Insect chromosome banding: technique for G- and Q-banding pattern in the mosquito Aedes albopictus. Can J Genet Cytol 1975, 17:241-244.

31. Jimenez LV, Kang BK, DeBruyn B, Lovin DD, Severson DW: Characterization of an Aedes aegypti bacterial artificial chromosome (BAC) library and chromosomal assignment of BAC clones for physical mapping quantitative trait loci that influence Plasmodium susceptibility. Insect Mol Biol 2004, 13:37-44.

32. Bosio CF, Fulton RE, Salasek ML, Beaty BJ, Black WC: Quantitative trait loci that control vector competence for dengue-2 virus in the mosquito Aedes aegypti. Genetics 2000, 156:687-698.

33. Gomez-Machorro C, Bennett KE, del del Lourdes Munoz M, Black WC: Quantitative trait loci affecting dengue midgut infection barriers in an advanced intercross line of Aedes aegypti. Insect Mol Biol 2004, 13:637-648

34. Severson DW, Mori A, Zhang Y, Christensen BM: Chromosomal mapping of two loci affecting filarial worm susceptibility in Aedes aegypti. Insect Mol Biol 1994, 3:67-72

35. Severson DW, Thathy V, Mori A, Zhang Y, Christensen BM: Restriction fragment length polymorphism mapping of quantitative trait loci for malaria parasite susceptibility in the mosquito Aedes aegypti. Genetics 1995, 139:1711-1717.

36. Zhong D, Menge DM, Temu EA, Chen H, Yan G: Amplified fragment length polymorphism mapping of quantitative trait loci for malaria parasite susceptibility in the yellow fever mosquito Aedes aegypti. Genetics 2006 173:1337-1345.

37. Brown SE, Knudson DL: FISH landmarks for Aedes aegypti chromosomes. Insect Mol Biol 1997, 6:197-202.

38. Smith CD, Shu S, Mungall CJ, Karpen GH: The Release 5.1 annotation of Drosophila melanogaster heterochromatin. Science 2007, 316:1586-1591.

39. Timoshevskiy VA, Sharma A, Sharakhov IV, Sharakhova MV: Fluorescent in situ hybridization on mitotic chromosomes of mosquitoes. J Vis Exp 2012, 67:e4215.

40. Newton ME, Southern DI, Wood RJ: X and Y chromosomes of Aedes aegypti (L.) distinguished by Giemsa C-banding. Chromosoma 1974, 49:41-49.

41. Sousa RC, Bicudo HEMC: Heterochromatic banding pattern in two Brazilian populations of Aedes aegypti. Genetica 1999, 105:93-99.

42. Hoskins RA, Carlson JW, Kennedy C, Acevedo D, Evans-Holm M, Frise E, Wan KH, Park S, Mendez-Lago M, Rossi F, Villasante A, Dimitri P, Karpen GH, Celniker SE: Sequence finishing and mapping of Drosophila melanogaster heterochromatin. Science 2007, 316:1625-1628.

43. Larson K, Yan SJ, Tsurumi A, Liu J, Zhou J, Gaur K, Guo D, Eickbush TH, Li WX: Heterochromatin formation promotes longevity and represses ribosomal RNA synthesis. PLoS Genet 2012, 8:e1002473.

44. Li H, Rodriguez J, Yoo Y, Shareef MM, Badugu R, Horabin Jl, Kellum R: Cooperative and antagonistic contributions of two heterochromatin proteins to transcriptional regulation of the Drosophila sex determination decision. PLOS Genet 2011, 7:e1002122.

45. Schaeffer SW, Bhutkar A, McAllister BF, Matsuda M, Matzkin LM, O'Grady PM, Rohde C, Valente VL, Aguade M, Anderson WW, Edwards K, Garcia AC, Goodman J, Hartigan J, Kataoka E, Lapoint RT, Lozovsky ER, Machado CA, Noor MA, Papaceit M, Reed LK, Richards S, Rieger TT, Russo SM, Sato H,
Segarra C, Smith DR, Smith TF, Strelets V, Tobari YN, et al: Polytene chromosomal maps of 11 Drosophila species: the order of genomic scaffolds inferred from genetic and physical maps. Genetics 2008, 179:1601-1655

46. Gonzalez J, Ranz JM, Ruiz A: Chromosomal elements evolve at different rates in the Drosophila genome. Genetics 2002, 161:1137-1154.

47. Caceres M, Ranz JM, Barbadilla A, Long M, Ruiz A: Generation of a widespread Drosophila inversion by a transposable element. Science 1999, 285:415-418.

48. Mathiopoulos KD, Della Torre A, Predazzi V, Petrarca V, Coluzzi M: Cloning of inversion breakpoints in the Anopheles gambiae complex traces a transposable element at the inversion junction. Proc Natl Acad Sci U S A 1998, 95:12444-12449.

49. Aulard S, Vaudin P, Ladeveze V, Chaminade N, Periquet G, Lemeunier F: Maintenance of a large pericentric inversion generated by the hobo transposable element in a transgenic line of Drosophila melanogaster. Heredity (Edinb) 2004, 92:151-155.

50. Lyttle TW, Haymer DS: The role of the transposable element hobo in the origin of endemic inversions in wild populations of Drosophila melanogaster. Genetica 1992, 86:113-126.

51. Lobachev KS, Rattray A, Narayanan V: Hairpin- and cruciform-mediated chromosome breakage: causes and consequences in eukaryotic cells. Front Biosci 2007, 12:4208-4220.

52. Vector Base. [http://www.vectorbase.org]

53. Haider S, Ballester B, Smedley D, Zhang J, Rice P, Kasprzyk A: BioMart Central Portal-unified access to biological data. Nucleic Acids Res 2009, 37:W23-W27.

54. Benson G: Tandem repeats finder: a program to analyze DNA sequences. Nucleic Acids Res 1999, 27:573-580.

55. Repeat Masker. [http://www.repeatmasker.org]

56. TEfam database. [http://tefam.biochem.vt.edu]

57. OrthoDB. [http://orthodb.org/orthodb7]

doi:10.1186/1741-7007-12-27

Cite this article as: Timoshevskiy et al:: Genomic composition and evolution of Aedes aegypti chromosomes revealed by the analysis of physically mapped supercontigs. BMC Biology 2014 12:27.

\section{Submit your next manuscript to BioMed Central and take full advantage of:}

- Convenient online submission

- Thorough peer review

- No space constraints or color figure charges

- Immediate publication on acceptance

- Inclusion in PubMed, CAS, Scopus and Google Scholar

- Research which is freely available for redistribution 